\title{
Information Quality Challenges of Patient-Generated Data in Clinical Practice
}

\author{
Peter West ${ }^{1 *}$, Max Van Kleek ${ }^{2}$, Richard Giordano ${ }^{1}$, Mark Weal $^{3}$ and Nigel Shadbolt ${ }^{2}$ \\ ${ }^{1}$ Faculty of Health Sciences, University of Southampton, Southampton, United Kingdom, ${ }^{2}$ Department of Computer \\ Science, University of Oxford, Oxford, United Kingdom, ${ }^{3}$ Web and Internet Science, Faculty of Physical Science and \\ Engineering, University of Southampton, Southampton, United Kingdom
}

A characteristic trend of digital health has been the dramatic increase in patient-generated data being presented to clinicians, which follows from the increased ubiquity of self-tracking practices by individuals, driven, in turn, by the proliferation of self-tracking tools and technologies. Such tools not only make self-tracking easier but also potentially more reliable by automating data collection, curation, and storage. While self-tracking practices themselves have been studied extensively in human-computer interaction literature, little work has yet looked at whether these patient-generated data might be able to support clinical processes, such as providing evidence for diagnoses, treatment monitoring, or postprocedure recovery, and how we can define information quality with respect to self-tracked data. In this article, we present the results of a literature review of empirical studies of self-tracking tools, in which we identify how clinicians perceive quality of information from such tools. In the studies, clinicians perceive several characteristics of information quality relating to accuracy and reliability, completeness, context, patient motivation, and representation. We discuss the issues these present in admitting self-tracked data as evidence for clinical decisions.

Keywords: self-tracking, quantified self, personalized medicine, information quality, health informatics, clinical decision making

\section{INTRODUCTION}

Apple, Google, Microsoft, Fitbit, Withings, and Nike are among dozens of brands in the fastgrowing consumer market of digital tools for keeping track of one's daily activities, fitness, health, and wellbeing (1). The proliferation of such devices has made the activity of self-tracking (2)-the collection of recording data about oneself-mainstream, by enabling such recording to be done with unprecedented granularity, at little effort or cost. While the first waves of such devices have focused on apps that help individuals achieve short-term health and fitness targets, potential longer term uses for such patient generated data created by such self-tracking practices have been proposed. In the UK, the recent Personalised Health and Care 2020 policy anticipated that by 2018 data generated by self-tracking products should start to become integrated with patient health records, allowing clinicians to act on the detailed data that patients record (3). This policy envisioned that patients contributing self-tracked data would be a key element toward improving healthcare quality and outcomes, while reducing costs. At the same time, patients would become empowered through use of this data, and have a better understanding of their own health. Literature on self-tracking, 
including those studying active participants of the Quantified Self self-tracking movement (4), have supported these arguments (5) and advocated for self-tracking as a way to reduce healthcare costs in monitoring chronic illness, while Swan et al. concluded that self-tracked data could serve a key role in preventative and precision medicine (6).

The Fitbit Surge is one such wearable self-tracking device, which automatically tracks heart rate, sleep patterns, physical activity, and distance moved. The device connects with a mobile app which displays self-tracked data as progress toward daily goals and plots over time. A substantial number of people own a Fitbit device, with 3.4 million sold in second quarter 2017 (7). Fitbit and other similar tools have made self-tracking accessible to the masses by simplifying the collection of data about health, and by presenting data in simple, easy-to-understand formats (8). Other popular products record a diverse array of information streams relating to health, such as the Apple Watch, which can record physical activity and sleep, the Caffeine Tracker app, which allows people to log how much caffeine they consume, and Daylio, an app for tracking ones mood over time.

The popularity of self-tracking has provoked interest for supporting clinical decisions, with self-tracked data "bridging the gaps" between clinical consultations and letting doctors build an accurate picture of patients over a long period of time $(2,9)$. These kinds of data are more diverse and detailed than traditional clinical measurements (10) and describe daily activity patterns (11) and first-hand descriptions of patient experience (12). Health tracking has been introduced into some workplaces to encourage health and wellness in employees (13). Automatic tracking through sensors, as opposed to manually recording activity, has been particularly successful at encouraging greater physical activity and health promotion (13). When collected on a population scale, data from wearable devices could provide clues to the causes of chronic conditions, such as the walkability of a city on the amount of physical activity of its citizens, which, in turn, could help shape public health policy (14).

However, several concerns have been raised about the quality of information from self-tracking practices. First, self-tracking devices have unknown reliability and validity, with most wearable device manufacturers providing no empirical evidence to demonstrate the effectiveness of their devices (15). Self-tracked data may be perceived as less reliable than medically proven lab data, and presenting self-tracked data with lab data could confuse clinicians about what data they can reliably use (16). This may be exacerbated by a high risk of patients' use of self-tracking tools lacking scientifically rigor (17). For example, there may be a high margin of error when patients without medical training try to associate symptoms to patterns in their data (15). It has been suggested that such tools could be designed to fail in conditions where patients are trying to form unscientific conclusions (17). Second, there may be different representations of data between different patients. Patients have different preferences for selftracking, and supporting these preferences lowers the burden for self-tracking. However, this may result in different data representations, which could create challenges for their integration into clinical care (18). Finally, the consistency of self-tracker use and abandonment may be challenges in the clinical use of self-tracked data. Users of trackers typically do not self-track on a daily basis, but take breaks every few days, and trying to force users to change this behavior will likely lead to abandonment (19). Development of frameworks for using self-tracking devices in healthcare could provoke suitable validation of data from such devices (15).

Were self-tracked data to be used in clinical decision-making, an essential threshold would have to be crossed: self-tracked data would have to be determined to be of sufficient quality to be of use in clinical decisions. In clinical settings, information quality is assured today through a multiplicity of measures, including protocols relating to methods of measurement, calibration and testing of instruments, and how the data are stored, retrieved, and used (20). In the context of patient-generated self-tracked data however, no such guarantees can be made. Self-tracking tools produce data which are new and unfamiliar to clinicians, and are seldom clinically validated (21). One study of pain management apps found that just $0.4 \%$ of apps had undergone clinical evaluation (21). How, then, can quality issues of information captured during self-tracking be resolved to make them suitable for clinical applications, such as in care delivery or personalized medicine?

This paper investigates the following research question, which we see as foundational to the clinical use of self-tracked data: how do we understand information quality in the context of selftracked data? By better understanding information quality, we can more usefully forecast how such self-tracked data can support clinical uses, as well as identify the challenges that remain before greater clinical adoption can be achieved. As such, we aim to address five core questions:

- How do perceived accuracy differences in self-tracking tools affect whether and how device data are used in workflows?

- What information quality issues have been discussed previously in the use of data of digital self-tracking and paper based diaries?

- What kinds of self-tracked data were perceived as potentially the most useful by clinicians, and what were information quality needs for each?

- Are needs pertaining to information quality the same for all uses and types?

- How and where (in workflows) are issues of self-tracking compliance and motivation most salient and incorporate consideration of factors that affect compliance?

To investigate this question, we sought to understand how doctors perceive the quality of information from self-tracking practices. We conducted a literature search and review, broadly inclusive of different kinds of self-tracking (both digital and paper based), scoped only to include empirical studies pertaining to data use by clinicians and other medical professionals. We found that existing empirical research addressing self-logged information quality in clinical use was scarce, mostly limited to specific chronic care contexts, such as irritable bowel syndrome (9), pain management (22), and heart failure (23). We analyzed these studies to identify the specific information quality issues encountered, and organized these into themes.

We found a common set of challenges around accuracy and reliability, completeness, context, patient motivation, and representation. We discuss implications that these themes have 
for the short- and long-term use of self-logged data in clinical practice.

\section{MATERIALS AND METHODS}

A survey of the literature on self-tracked data was conducted to analyze the current knowledge on clinical use of self-tracked data. We use the Preferred Reporting Items for Systematic Reviews and Meta-Analysis (PRISMA), which is an evidence-based minimum set of items for reporting systematic reviews or meta-analyses (24) as an audit trail of selected articles. See Figure 1 for the PRISMA flow diagram of this literature search.

\subsection{Search Strategy}

Searches were conducted on JSTOR, EBSCOhost (MEDLINE), PubMed, Cochrane, Web of Science, Scopus, and ACM DL databases (see Table 1). These databases were chosen because they index high quality journals, and encompass a multidisciplinary knowledge base. A search strategy was constructed to find literature relating to self-tracking in clinical settings:

"patient " AND ("clinic ${ }^{\star *}$ OR "provider*” OR "doctor*” OR “* care professional*” OR "ambulatory") AND ("self track*” OR "activity track*” OR "life log*” OR "lifelog*” OR "quantified self" OR "quantified patient" OR "patient generated" OR "consumer health information tech"” OR "consumer wearable*" OR "personal informatics" OR ("self monitoring” AND “diary"))

Because the phrase self-tracked data has many synonyms and related terms (including life-logging, quantified self, and patient-generated information), the search query was expanded to include these terms. The term self-monitoring generated a vast number of results relating to prescribed practices and clinical devices unrelated to self-tracking, hence diary was added to limit results to patients self-tracking their health. Wildcards were used in the search query to match similar words (for example, track matches both tracked and tracking, and Boolean operators were used to constrain results to those with a sufficient set of relevant terms. All databases supported wildcards and Boolean operators, though some required the query to be split over multiple searches.

\subsection{Selection Criteria}

To focus on clinicians' perceptions on information quality issues, we focused only on studies in which medical experts (comprising doctors, nurses, specialists, and others from primary and secondary care) accessed, used, or otherwise interacted with patient generated data (PGD). Because we were not assessing the clinical effects per se of self-tracking or the use self-tracked data, we did not use a standard appraisal tool, such as CASP, or a formal published checklist when selecting the papers (25). Inclusion was based on three criteria. First, the article must have

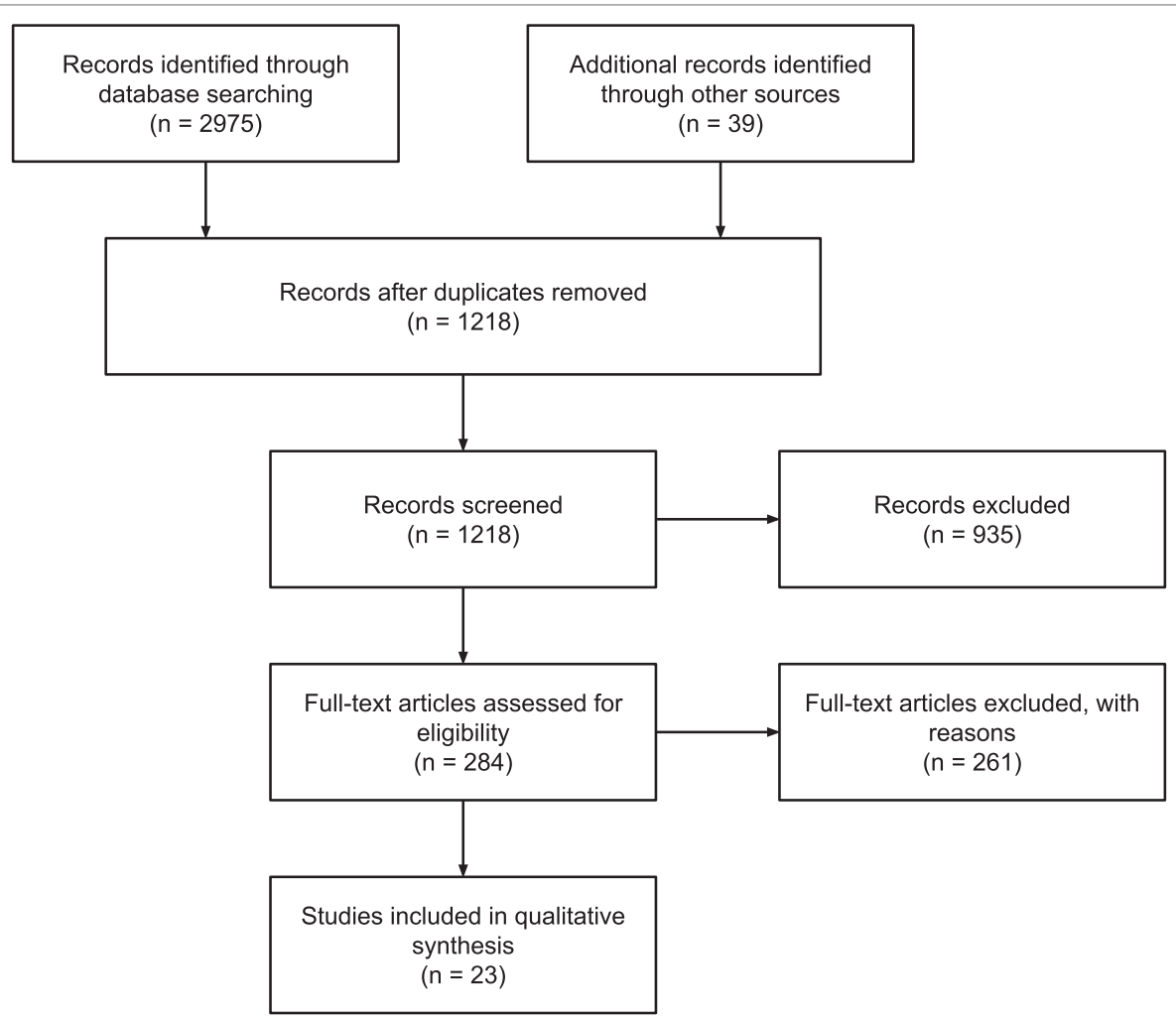

FIGURE 1 | PRISMA flow diagram of the literature review. 
TABLE 1 | Search strategy.

\begin{tabular}{llc}
\hline Database & Discipline & Records \\
\hline JSTOR & Multidisciplinary & 109 \\
EBSCOhost (MEDLINE) & Medicine & 366 \\
PubMed & Medicine & 504 \\
Cochrane & Medicine & 1 \\
Web of Science & Multidisciplinary & 321 \\
Scopus & Multidisciplinary & 711 \\
ACM DL & Computing & 39 \\
\hline Total & & 2,975 \\
Total (without duplicates) & & 1,218 \\
\hline
\end{tabular}

been peer-reviewed, and represent empirical work, thus excluding opinion, literature surveys, and self-experimentation pieces. Second, the subject matter must have pertained to data collection by the patient using non-clinical tools. This included consumer devices, paper diaries, and patient-facing portals, but excluded telemonitoring, implantables, and other forms of data or technology used in clinical settings. Studies relating to patient-reported outcomes were also excluded, as these forms of data were usually retrospective forms of information gathering (26), rather than pervasive self-tracking. Third, the findings had to pertain to how self-tracked data were used by clinicians, including how information quality was evaluated, and used in decision-making. This excluded papers solely concerned with self-tracking for selfreflection or self-improvement. Studies about using self-tracking for research, or "big data" uses, and reviews of efficacy of selftracking techniques were excluded as these did not relate to a clinician's perceptions of information quality.

Several papers which were not present in the search results but were known to the authors were also included. In total, twentythree papers met the inclusion criteria. Of the papers excluded, 996 did not pertain to patient self-tracking, 129 did not consider clinician's perspective, 55 were not empirical, 9 were not peerreviewed articles, 4 concerned big data only, and 4 were studies similar to other papers in the selection.

\subsection{Analysis}

Papers were divided and read through by three members of the research team to identify and tabulate clinical setting, study rationale, methods, and key conclusions from each article on selftracking in clinical settings. Three members of the team derived themes related to information quality based on the conclusions of the articles. These themes were refined by all team members.

\section{RESULTS}

Twenty-three papers resulted from our literature search; these are summarized in Table 2. Within the literature, we identified six themes relating to information quality of self-tracked data, which are listed in Table 3 with the papers they were identified within. In this section, we describe each of the six themes in order of their prominence within the literature.

\subsection{Structure and Presentation}

The most prominent theme deriving from our analysis pertained to the ways that self-tracked data were structured and represented. Clinical information systems, including EMRs, as well as diagnostic devices and systems, represent patient data in largely standardized ways. Such standardized representations help clinicians efficiently and accurately interpret data (9), even when such data are multivariate and voluminous. Self-tracking tools, however, were found to rarely, if ever, use or derive representations based on these standards. Several reasons have been posited for this disparity; one, for example, that most self-tracking tools are developed by tech companies with little expertise in clinical informatics. Moreover, these apps are typically created for consumers, and not for clinical purposes. Other plausible reasons are that these apps are deliberately designed to be simple, easy-to-use, and to avoid representations that could be perceived as potentially too technical in order to appeal to non-specialist individuals (9).

Beyond being unlike clinical representations, there appears to be significant variation across different self-tracking tools, whether wearable sensors $(30,33,34)$, or electronic self-tracking apps $(9,22,23,31-33,36,37,41,47)$. Such variations extended beyond the specific visual representations used to present and summarize the data, to variations of data granularity, aggregation methods, to units of measure. When patients used more traditional methods, such as keeping hand-written notes, or using general-purpose tools such as word processors or spreadsheet tools, the result was often similarly varied but for different reasons; patients were seen to naturally structure their data in ways most intuitive to them, which was often idiosyncratic to their preferences and goals. Both kinds of variations and disparities of representation were seen as a direct and primary obstacle to quick, safe, and effective use of self-tracked data.

The relative importance of the use of specific representations also depended on the role of the expert reviewing the data. Primary care physicians and nurses were often more flexible in terms of "piecing together" disparate evidence, based on heterogeneous, varied information sources, including data from self-tracked tools and recounted personal experience (44). Secondary care specialists, however, more often expressed the need to reorganize, reorder, and sometimes restructure information into standardized forms (such as the clinical admissions form) before being able to effectively evaluate it (44).

The nature and kinds of relationships sought in the data often shaped the representations that were seen as most helpful. In one study, clinicians said that temporal relationships were the most important, as they established causal relationships between potential triggers and symptoms. As a result, timelines were seen as the most natural way to represent patient self-tracked diaries (35). In such timelines, both ordering (indicating precedence) and temporal distance were viewed as important for suggesting or ruling out causal links; for example, a symptom occurring before, or too long after a potential cause would suggest the two unrelated, and a timeline would make such connections quickly identifiable (44).

Typically, clinicians were unclear on how self-tracked data could be effectively presented, primarily because the data describe unfamiliar forms of measurement, such as sensor data from an accelerate. Clinicians are responding to self-tracking practices as new forms of data collection that are unfamiliar. However, as these practices and tools evolve, it is likely that the 
TABLE 2 | Empirical studies in clinical settings, listing the overview, method, and findings pertaining to information quality of each study.

\begin{tabular}{ll}
\hline Reference & Study overview \\
\hline$(27,28)$ & Clinicians often lack complete or \\
& accurate information about patients \\
& with multiple chronic conditions \\
& (MCC) leading to poor care \\
& coordination and medical errors
\end{tabular}

(29) Migraine often undertreated due to impaired clinician-patient communication and clinicians underestimate migraine severity

(30) Management of weight loss is difficult, leading to underdiagnosis and undertreatment

(9) Patients with irritable bowel syndrome (IBS) often self-track lifestyle changes are often dissatisfied with feedback from clinicians despite its potential to improve efficacy of behavior change efforts

(31) Builds on (9)

(32) Builds on (9)

\section{Method}

Interviews 7 MCC clinicians and 22 MCC patients about self-tracking of health-related information (e.g., test results, medications) using paper or electronic tools

\section{Findings}

Problems arise from difficulty of self-tracked data retrieval, perceived emotional valence of data, concern that the patient seems obsessive, and concern that data ma be selective reported (e.g., to avoid insurance premiums). Clinicians pursue clinical measurements over self-tracked data, though data of any form is preferable to none; patient information allows best decisions over care

Questionnaire of 118 patients and 22 clinicians about clinician-initiated self-tracking using paper diary with questionnaires about pain, disability, and medication

Self-tracked data improves patient-clinician communication and increases patient satisfaction, possibly due to more time being spent with patient. It enables assessment of pain intensity and disability and subsequent prescription of medications

Analysis of 30 patients following 1 month use of a clinician-initiated self-tracking app and wearable sensors

Only a few participants shared data with doctors, despite this being a feature of the app. App usage negatively impacted patient-doctor relationship

Twenty-one clinicians who work with IBS patients interviewed about their current and potential uses of patient-initiated self-tracked information from paper or electronic tools

Self-tracking tools lack flexibility and standardized formats, and doctors have a lack of time and skills to interpret such data. Nonetheless, it helps understand IBS patients' routines and enhances clinician-patient communication and personalization of treatment plans. Contextual information helps better understand the patient. For, diagnosis high reliability and granularity is required

For collaboration with patient, clinicians create "compilation artifacts" from self-tracked data by collecting information from different sources, organizing it, and presenting it. Some clinicians prefer paper for better interaction affordances. It is important to highlight missing data or mistakes for accountability patients, and reanalysis of 21 clinician interviews from Ref. (9) about self-tracking of health-related information using paper or electronic tools

Doctors wanted to know how patient data compares to Interviews of $10 \mathrm{IBS}$ patients and 10 clinicians who work with IBS patients. Clinician-initiated self-tracking of food and symptoms using bespoke mobile app population-level data. Clinicians trust patients to interpret their own data during visits to support communication. Contextual information is important for trust, though doctors lacked trust in patient's judgment of symptom severity. Data can support hypothesis generation on what may be causing a patient's symptoms

\begin{tabular}{ll}
\hline (33) Support self-management for \\
healthy eating and preventing and \\
managing disease
\end{tabular}

Interviews of 13 study designers and 12 healthcare professionals. Self-initiated and Self-tracked data provides a deep and accurate insight into patient's condition between clinic visits of apps, sensors, and websites

(23) Rehospitalization is a common occurrence for patients with heart failure (HF); preventing rehospitalization could reduce costs and decrease mortality

Observation of $124 \mathrm{HF}$ patients using a diary

Diary-taking encourages more frequent contact with clinicians. for 6 months and analysis of clinical outcomes. Early reporting of health changes can strengthen program effect Clinician-initiated self-tracking using paper or computerized diary for recording weight, symptoms, and any other behavior patients considered relevant

(34) Self-management using a Fitbit could encourage lifestyle changes which prevent chronic disease Seventeen people at risk of chronic disease from obesity completed a study of selfmonitoring using fitness trackers and perceived quality of life. Mandated self-tracking using fitness trackers and patient surveys

(35) Patients not only want access to various medical records their health care providers keep about them but also are willing to become active participants in managing their health information Personal health records (PHRs) were developed to help fulfill this need. There is little understanding of different health care practitioners' views of PHRs, including how PHRs could fit into the existing health care system, is lacking
Twenty-one clinical practitioners with 10 different specialties were interviewed. Semistructured interviews where participants were asked to describe an "ideal" personal health record
Self-tracking shifts responsibility of health to the patient and helps alleviate time constraints of primary care physicians

History must be presented chronologically. Different types of information are more important to some clinicians (e.g., Chinese medicine) than others. Charts and time lines are critical 
TABLE 2 | Continued

\begin{tabular}{ll}
\hline Reference & Study overview \\
\hline (36) & $\begin{array}{l}\text { Breast cancer patients often have } \\
\text { difficulty managing their own health } \\
\text { information due to changes in } \\
\text { health and goals over time }\end{array}$ \\
\hline (37) & $\begin{array}{l}\text { Self-tracking may help in prevention } \\
\text { and management of lifestyle } \\
\text { diseases }\end{array}$
\end{tabular}

\section{Method}

Interviews of 12 breast cancer patients.

Clinician-initiated self-tracking using tablet with Note taker, MyFitnessPal, and other cancer apps

Observation of 20 lifestyle disease patients and 6 specialists with self-tracked food logs, steps, and sleeping time from a mobile app
(22) People with chronic illness may be able to better self-manage using self-tracking

\section{(38) Hospitalized patients may have a} better experience and improved health outcomes if they are treated as stakeholders in their care and if clinicians have better access to health information
Interviews of 12 chronic illness patients who self-track. Self-initiated self-tracking using apps and paper to track episodes, triggers, medication, status, and history

Twenty-eight inpatients and their caregivers were interviewed and observed at bedside to understand their interactions with clinicians. Self-initiated self-tracking of various forms

\section{Findings}

Self-tracking supports communication with doctors because patients feel more prepared and confident; patient feels empowered. Mobility of the tool allows patients to readily retrieve information and questions for the doctor

Self-tracking enables more thorough history taking. Raw data required for additional analysis, and visualizations or summaries for "at a glance." Clinicians expect low adherence rate and don't have confidence in the accuracy of self-tracked data. Clinicians overlap self-tracked data with other data (e.g., sleep onto calorie intake). Although this isn't usually enough evidence to determine cause and effect, it helps understand the patient by showing context

Self-tracked data facilitates effective doctor-patient communication and identification of immediate specific needs and triggers of episodes. For poorly understood conditions, self-tracked data may be the only factor for deciding how to manage their condition

Self-tracking may prevent medical errors by providing information
when the clinician doesn't have it
(39) Mobile health and patient-generated Twelve primary care physicians and health data are promising health IT endocrinologists. Individual interviews and tools for delivering self-management survey support in diabetes, but little is known about provider perspectives on how best to integrate these programs into routine care. Provider perceptions of a patient-generated health data report from a textmessage-based diabetes selfmanagement program are explored

(40) Breast cancer patients often have difficulty communicating symptoms to clinicians

(41) This paper discusses mediation in the patient-provider relationship arising from the introduction of digital technology for a specific form of monitoring: "clinical self-tracking"

Twenty-one diabetes 1 patients; 4 doctors, 8
(46) Paper food and gastrointestinal symptom journals used to help irritable bowel syndrome (IBS) patients determine potential trigger foods. The study evaluated the feasibility, usability, and clinical utility of such journals as a data collection tool, and explore a method for analyzing journal data to describe patterns of diet and symptoms
Twenty-five breast cancer patients were interviewed about self-tracking and observed at home and in clinical visits. Self-initiated selftracking of symptoms using bespoke mobile app or patient's own technique parents, and 2 nurses at three clinics in Italy. $A$ smartphone application enabled patients to keep track of all the information relative to their diabetes; web-based dashboard accessible by doctors with a system of rule-based alarms designed to send an alert to clinicians and/or patients in the presence of certain data or combinations of data, and a messaging platform that worked as a secure email service between patients was developed and trialed over 3 months. Interviews with respondents to evaluate it

Caucasian males $(\mathrm{N}=13)$ women $(\mathrm{N}=14)$ Mean age $35 \pm 12$. Participants logged three sets of 3-day food and symptom paperbased journals over a 15-day period. Subjects participated in follow-up interviews
Self-tracking supports communication with clinicians by helping patient's recall events and providing a basis for prioritizing symptoms. Support patient reflection and doctor-patient communication by allowing overlapping of graphs to see co-occurrences of symptoms and events. Enable clinicians to explain salient parts of the data to patients so they can understand what is happening

Respondents reported that data were more reliable from a smartphone than from a clinic visit because patents were less willing to "please the doctor." Data were useful in understanding the patient, developing a focus in a treatment plan, increasing patient engagement, and understanding the patient's perspective. Self-generated data did not directly influence care, but instead enhanced it

Adolescents and adults with poorly controlled diabetes did not share data with providers; the level of autonomy is different based on patient type (e.g., children, pregnant women). Intended users of the system are not presented with a binary choice between use and non-use; rather, they enact technology selectively to fit into their lives. Authors suggest that "pushing" self-tracking by the clinician might be perceived by patients as being intrusive

Over half perceived paper journaling of food and symptoms as feasible, usable, and clinically useful. Thirteen participants demonstrated a strong association with at least one symptom and meal nutrient. No mechanism to capture time of completion or accuracy of entries. Journal entries for IBS patients are shown to be feasible, usable, and have clinical utility. Paper-based entries have weaknesses related to accuracy and veracity since there are no automatic mechanisms to check for these. IBS patients are possibly more motivated than others to complete entries 
TABLE 2 | Continued

\begin{tabular}{|c|c|c|c|}
\hline Reference & Study overview & Method & Findings \\
\hline (42) & $\begin{array}{l}\text { Consequences of personal health } \\
\text { records on the health care system } \\
\text { are poorly understood, in particular } \\
\text { the temporal and cognitive burden } \\
\text { associated with new workflows that } \\
\text { include PGD. This paper reports the } \\
\text { results for time-cost and resource } \\
\text { utilization of a "typical" ambulatory } \\
\text { clinic under varying conditions of } \\
\text { PGD burden }\end{array}$ & $\begin{array}{l}\text { The time-cost impact of patient-generated } \\
\text { data is measured using discrete event model } \\
\text { (DEM) simulation. Three simulation scenarios } \\
\text { of everincreasing PGD impact are compared } \\
\text { to a baseline case of no PGD use. A simulation } \\
\text { with doctor, nurse, support staff roles and their } \\
\text { costs. Real clinicians were used to estimate } \\
\text { time, and to generate a range of assumptions } \\
\text { that encompassed their estimates. The model } \\
\text { was validated by subject matter experts who } \\
\text { found that the simulator behavior they observed } \\
\text { was consistent with clinic operation as they } \\
\text { were accustomed to it }\end{array}$ & $\begin{array}{l}\text { There are close ties between PGD use and resource utilization, } \\
\text { including clinic layout and workflow design. Lengths of both } \\
\text { workday and patient visit were extended and less predictable } \\
\text { with PGD use. The integration of PGD in clinical work flows needs } \\
\text { extensive preparation because the impact of PGD use is non-trivial } \\
\text { and will quantifiably either cause longer workdays or mandate } \\
\text { the sacrifice of other activity to reap any argued or measureable } \\
\text { benefit }\end{array}$ \\
\hline (44) & $\begin{array}{l}\text { Decisions in diagnostic settings } \\
\text { must often be made with very little } \\
\text { information. Self-tracked data may } \\
\text { be useful as evidence in diagnoses }\end{array}$ & $\begin{array}{l}\text { Ten primary and secondary care physicians } \\
\text { from UK and USA role-played two diagnosis } \\
\text { scenarios relating to migraines and heart } \\
\text { conditions. While participants saw potential in } \\
\text { self-tracked data being used as evidence, in } \\
\text { practice data were considered untrustworthy } \\
\text { due to unknown patient motivations and unclear } \\
\text { reliability of the recording device and technique }\end{array}$ & $\begin{array}{l}\text { Self-tracked data in diagnostic settings may be dismissed as } \\
\text { unreliable or untrustworthy. A patient's motivation for self-tracking, } \\
\text { the routine they followed to take measurements, and the device } \\
\text { they used influence a doctors decision to use the data }\end{array}$ \\
\hline (43) & $\begin{array}{l}\text { Long-term measurements in a } \\
\text { home environment could support } \\
\text { screening and medical treatment. } \\
\text { This paper describes considerations } \\
\text { and recommendations for the } \\
\text { design of sleep monitoring tools }\end{array}$ & $\begin{array}{l}\text { Observations of } 8 \text { staff at two clinical sleep } \\
\text { centers in Belgium, and } 1 \text { ambulatory sleep } \\
\text { center in the Netherlands while preparing, } \\
\text { executing, and processing sleep studies. Notes } \\
\text { were translated to an affinity diagram that was } \\
\text { used to uncover patterns in the interviews and } \\
\text { observations. No community self-tracking; } \\
\text { polysomnography in clinical settings }\end{array}$ & $\begin{array}{l}\text { Doctors want the raw data to analyze for themselves. Self- } \\
\text { monitoring sleep tools should fit in existing hardware and software, } \\
\text { using existing standards. Sleep clinicians depend, in part, on video } \\
\text { when monitoring sleep. Patient collected data need to contain } \\
\text { context that substitutes for video }\end{array}$ \\
\hline$(45)$ & $\begin{array}{l}\text { Patients are increasingly tracking } \\
\text { and generating an increasingly large } \\
\text { volume of personal health data } \\
\text { from wearable sensing and mobile } \\
\text { health (mHealth) apps, and that the } \\
\text { potential usefulness of these data } \\
\text { is enormous. This study explores } \\
\text { how patients and clinicians currently } \\
\text { share patient-generated data in } \\
\text { clinical care practice }\end{array}$ & $\begin{array}{l}\text { Twenty-one participants (12 patient participants } \\
\text { and } 9 \text { clinician participants). None directly. } \\
\text { Sample population used both clinically directed } \\
\text { and self-directed tracking. Results derived } \\
\text { from telephone, Skype, and face-to-face } \\
\text { semistructured interviews from a purposive } \\
\text { sample of patients and clinicians. Interviews } \\
\text { were coded by two researchers }\end{array}$ & $\begin{array}{l}\text { Doctor directed self-tracking motivated by desires to increase } \\
\text { patient engagement; assessing a condition over time; ascertain } \\
\text { the effect of lifestyle changes on health; belief that clinician } \\
\text { authority motivates patients to track. Clinicians cannot effectively } \\
\text { use patient generated data until it is integrated into the clinical } \\
\text { systems. Clinicians need to be incentivized to incorporate patient } \\
\text { generated into their everyday work flows. Clinicians need to } \\
\text { adapt to the cultural shift in healthcare, in which more patients are } \\
\text { attempting to make healthcare a collaborative endeavor }\end{array}$ \\
\hline
\end{tabular}

perspectives of clinicians will change, and an understanding of the next set of challenges for presenting information will become clear.

\subsection{Trustworthiness}

The term trustworthiness is often used in qualitative analysis (48) to describe the degree to which one can "gain knowledge and understanding of the nature of the phenomenon under study" (49). This is preferred to terms such as accuracy or reliability which describe a specific instrument's ability to measure the phenomenon (50). These aspects of trustworthiness were often perceived by clinicians as an aspect of information quality. We appropriate the term to reflect the broad set of concerns expressed toward gaining an effective understanding of the patient through their self-tracked data. This definition is left intentionally broad to be able to accommodate the particular concerns relating to distinct uses reported across the literature, ranging from recollection of symptoms and potential triggers $(23,29,32,47)$, to helping clinicians identify long-term trends $(22,23,43)$, to understanding symptomatic burden and impact on patients' overall quality of life (36).

For differential diagnosis, trustworthiness often corresponded to quantitative measures of data quality. This included accuracy, such as whether the devices and sensors used by patients to self-measure were accurate (51), as well as reliability, such as for self-report measures in health diary apps. For the former, considerations included the type of devices used take measurements, whether the devices were clinically calibrated, and whether they used sensing approaches analogous to those used by clinical instruments (31). Other concerns included issues of data sampling, including representativeness, and completeness of data, which depended on such as issues whether the data were recorded automatically or manually, and patients' ability and willingness to do so (45). Such factors that influenced trustworthiness included the duration over which data were logged (35), as well as granularity (9). When patient compliance with data collection was sometimes poor, resulting in gaps or insufficient detail, these 


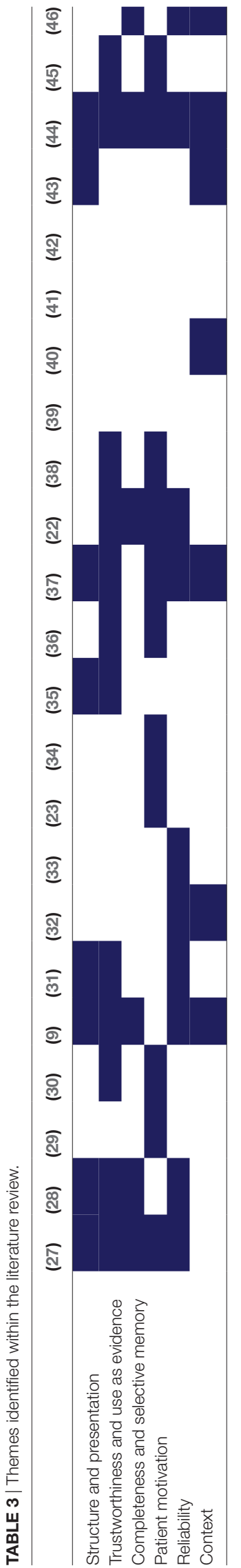

were seen as negatively impacting perceived trustworthiness (37). Moreover, clinicians feared that poor compliance was potentially indicative of selective reporting, which was seen as a means of concealing information from the clinician (27).

Across the literature surveyed, self-tracked data were, in general, considered less trustworthy than corresponding clinical data. This affected how self-tracked data were treated when used at various stages within workflows. For example, in differential diagnosis, prognostic decisions based on self-tracked data were made only after considering substantial additional supporting evidence, which was systematically sought (27). The most common approach to obtain more trustworthy evidence was running additional clinical diagnostics, which was done routinely to support hypotheses whenever it was practical $(27,44)$.

It is important to note, however, that trustworthiness was not always an important consideration. For example, at the point in a diagnostic process that all working hypotheses are ruled out, the opportunity arises to re-consider all available information toward new hypothesis formation (44). For this purpose, it was perceived valuable to consider all available information as potential evidence, regardless of perceived trustworthiness (27). In addition, when data were examined cooperatively with patients, such as for reflection (31) or to facilitate self-recall $(38,40)$, notions of trustworthiness of the data were less important than the personal significance and communicative roles served by the data. For such uses, instead of seeing self-tracked data as an extension of clinical data, e.g., as an evidential basis upon which to make clinical decisions, are more appropriately thought of as "boundary objects" through which the patient and clinician can achieve a shared understanding of the patient's condition (32).

\subsection{Patient Motivations}

Understanding the patient's motivations has been long discussed as a key challenge in effectively addressing patient concerns and delivering appropriate care $(52,53)$. The motivations of the patient, and therefore the patient's behaviors toward data collection, were perceived by clinicians to be an aspect of data quality. In the context of patient-generated data, understanding the patient's reasons for (as well as for not) self-tracking were sometimes considered in the process of understanding the patient's condition. For instance, the act of avid self-logging was sometimes seen as an indicator of obsession, compulsiveness, or significant concern about particular symptoms $(28,44,45)$. On the other hand, a lack of self-logging compliance, or ability to keep track of symptoms was seen as potential signs of apathy (54).

Sometimes, the act of presenting data to clinicians was seen to indicate some explicit underlying motivation, such as the desire to confirm a diagnosis, or worse, "begging for a diagnosis." Sometimes, the presentation of information was seen as a workaround for the inability to communicate something difficult to put into words. Usually, however, the presentation of PGD was simply an artifact of the fact that self-tracking was on the rise, and that patients felt such data would be useful for clinicians during the consultation $(9,44)$.

In the context of long-term health management, the motivations for self-tracking usually were unambiguous, relating to chronicling of symptom burden and other issues of concern to 
them $(9,23,37)$. When managing multiple chronic conditions, however, self-logging was often not perceived to be a reliable indicator because it was seen as both an excessive burden for patients who had already experienced significant disease burdens that limited their daily activities (27). Moreover, the lack of an evidence base for combinations of symptoms those with multiple chronic conditions, meant that logs for these patients were more ambiguous to interpret, and more difficult to translate to effective outcomes, and thus were seen as less of direct value to the patient.

\subsection{Capture Context and Metadata}

Contextual aspects of how self-tracked data are captured, such as location, time of day, activity, and posture, are important for understanding cause and effect where multiple medical conditions exist (43) and incomplete information contributes to medical errors (27). Single data streams on their own may provide insufficient context about a patient's recordings for clinicians to make suitable judgments from them. Self-tracking devices today rarely capture such contextual dimensions, and typically only capture limited metadata about measurements (55); self-tracking tools tend to focus on a single facet of people's lives (56) and users are not aware of important information that they do not collect (57). However, self-tracking devices, such as wearable sensors, provide a means of capturing data at high-resolution and granularity with little or no effort to end-users, supporting creation of time series datasets with a high degree of completeness (9). Future technology may be capable to automatically collating information from separate data sources to provide contextual information about a patient's recordings.

\subsection{Format and Representation}

The choice of format affects the clinician's ability both to interpret the data and trust it (32). The availability of contextual information (such as what the patient was doing and where they were during a measurement) is also a factor in trust (32) and contributes a better understanding of the patient (9). Contextual information could consist of other data sources available in a patient's health record or through self-tracking other kinds of information (32). Overlaying population-level data onto data visualizations may help in interpreting types of measurement uncommon in clinical settings $(32,40)$.

Healthcare information systems often do not provide ways to interoperate with self-tracked data $(9,27,37)$, and data which is entered into these systems are prone to getting lost (38). Thus, doctors are pragmatic in how data can be retrieved, with any data being better than none (27). Woods et al. (58) note that clinicians are more likely to accept types of data that fit a biomedical model. Similarly in constructing a risk model of integrating clinical and Quantified Self data, Third et al. (59) noted the need to adopt commonly used standards to aid integration of the data.

\section{DISCUSSION}

In this section, we bring together the themes identified in our literature review to address the question posed in the introduction: how do we understand information quality, in the context of self-tracked data?

\subsection{Synthesis: Dimensions of Self-Tracked Information Quality}

Information quality issues were seen to affect the ways self-tracked data were considered by clinicians during a consultation in many ways. Drawing on the themes from the literature review, we synthesized a view of these factors, visible in Figure 2, as a journey from the patient's initial collection of data, to use in clinical settings.

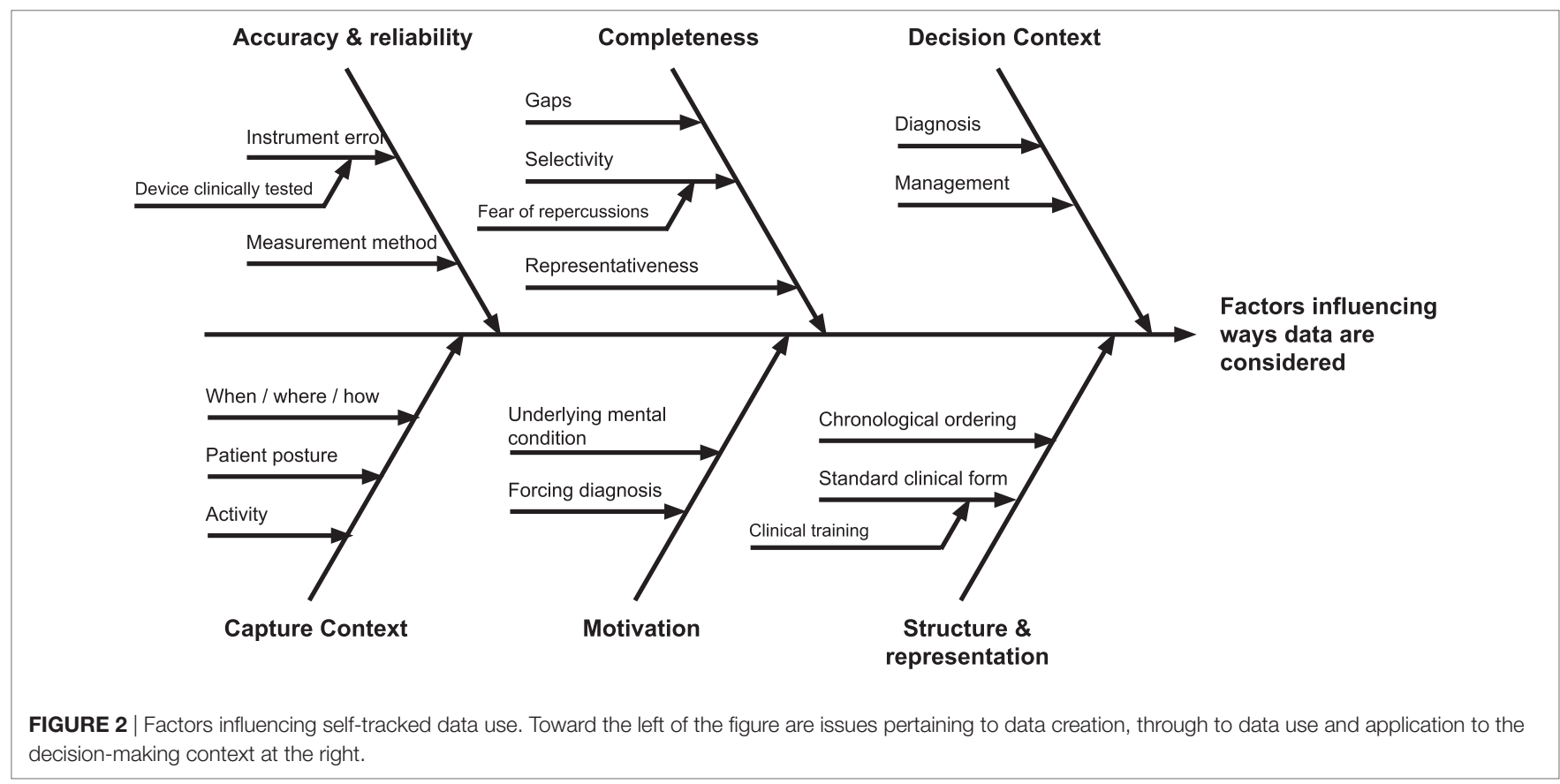


The most prominent theme primarily derived from our analysis was the importance of the decision context when interpreting important quality issues in self-tracked data. In particular, a context of differential diagnosis presented different quality needs and considerations for self-tracked data than the context of chronic condition self-management. For diagnosis, self-tracked could be used as supporting evidence in the service of deriving an appropriate plan of care, and thus establishing its veracity, provenance, and reflection of actual patient experience was viewed as crucial. For chronic condition management, however, literature suggests self-tracked data as primarily indicative of symptomatic burden and subjective patient experience, including quality of life.

A second prominent theme pertained to how self-tracked data were presented. It was clear from the literature that clinicians preferred patient data to be presented in particular ways, such as events ordered chronologically, or organized according to a familiar structure such as the ubiquitous hospital admissions record. It was found that patient-facing apps rarely provided presentation capabilities (such as statistical interpretation aids) on a par with those of clinical systems, because they were assumed to be used by non-specialists. As a result, underlying data quality issues aside self-tracking systems today were viewed as more time-consuming and difficult to interpret, merely from a lack of presentational support for effective interrogation.

On the far left of Figure $\mathbf{2}$ are information quality issues introduced at the creation of self-tracked data. This includes lowlevel information quality issues identified in the literature review pertaining to individual measurements, including accuracy and reliability, stemming from causes such as instrumentation error and incorrect methods of capture. Understanding how the measurements were taken (which were thematically described as capture context) including the patient's posture, activity immediately prior to, and when, where, and how data were taken, were discussed as a potential mechanism of enabling the assessment of reliability of self-made measurements, in the absence of controls for how they were taken.

Beyond individual measurements were quality issues of aggregated collections of self-tracked data, including longitudinal time-series measurements. For such collections, issues of data completeness, and sampling arose, including interpretations of gaps in important data, and representativeness of collected data. Understanding the latent causes were discussed for each of these, including, most significantly, underlying patient motivations in both self-tracking and presentation. It was deemed that such motivations, including "begging for a diagnosis," or wanting confirmation of a disease were important to consider as they were seen to naturally shape (and potentially bias) the data that patients would be inclined to present and emphasize. On the other hand, absence of data could be an indicate a lack of motivation or a lack of need to self-track, which could be interpreted either as being well or having other priorities.

\subsection{Applying the Factors: Scenario Walk- Through}

The results from the literature review now affords us the opportunity to construct a walk-through that delineates how the quality of self-tracked data, and the context of decision-making, affect the trustworthiness of information presented to a clinician. The purpose of this fictional multipart scenario, which is not based on real cases by medical experts, is purely to illustrate how the characteristics of information quality discussed in the previous section (see Figure 2) might manifest at various stages of a patient's clinical workflow.

Let us imagine that we have a patient, Rupert, who suffers chest pains when he climbs a flight of stairs. Rupert then records his blood pressure and heart rate and brings this information to his doctor. Working from the left-hand side of Figure 2, to the right, we will uncover the story of how and to what extent Rupert's data are trusted and used by the doctor. Given our model, Rupert's doctor will be concerned with the context by which Rupert captured the data, how accurate and complete the readings are, and what motivated Rupert to collect those readings. The doctor will also want to see the readings in a format that is understandable and usable. Is Rupert looking for a diagnosis of his symptoms, or is he visiting the clinic as part of a pathway to evaluate and assess a treatment plan?

\subsubsection{Diagnosing His Condition}

When Rupert presents his blood pressure and heart rate readings to his doctor, one of the first questions that will concern the doctor is how were the data collected? What was Rupert's posture when he took his readings? How long after climbing the stairs did Rupert take his measurement? Rupert might not remember this accurately; would the doctor simply take Rupert's word? This raises some questions in the doctor's mind about the context in which the readings were collected; consequently, the data is suspect.

Which instrument did Rupert use? How does Rupert's doctor know if this instrument is accurate? The doctor has a reading, but is this complete? Is it just a single reading? Is it an average of three readings? If it is an average, how much time passed between readings? Did Rupert use the device correctly?

How complete is this data to make a diagnosis? How can a simple blood pressure/heart rate reading diagnose heart disease? What gaps are there that need to be addressed to support a diagnostic judgment? Does Rupert have angina? Has he suffered a mild heart attack? Is it an esophageal spasm?

The doctor may then ask if Rupert is suffering from stress, and both the chest pains along with the data collection are merely symptoms of this.

Finally, is the data presented in a way that Rupert's doctor can understand-are they presented in a standard format? Is it a graph? Do the readings show actual values or just a graphical rendition of them?

If Rupert expected that the doctor would use his data to support a diagnosis, he would be disappointed because Rupert's doctor cannot trust this data to support a diagnosis. Instead, the doctor may glance at Rupert's data out of politeness, set it aside, and decide on which tests he would order to support a diagnosis, or refer Rupert to a cardiologist.

\subsubsection{Managing His Condition}

In this scenario, let us assume that Rupert has already been diagnosed with angina, has been prescribed medication, and his 
doctor is evaluating a treatment plan. Part of that plan is to visit the clinic with weekly blood pressure readings, heart rates, and activity logs. In this scenario, Rupert's blood pressure device has been calibrated with a device in the doctor's office, and Rupert is wearing a wearable device recommended by his doctor that logs activity and measures his heart rate. Rupert has been instructed on when and how to measure his blood pressure.

The doctor is satisfied with the context of data capture, the accuracy of the readings, and the completeness of the data. Rupert's doctor is also satisfied that Rupert is not a member of the "worried well," but someone who desires to manage his heart condition. The devices that the doctor has instructed Rupert to use display information in a manner that is useful to the doctor, and, as a bonus, can be transferred to Rupert's electronic medical record. The data, while still not complete enough to support a diagnosis, is of high enough quality to document Rupert's condition between clinic visits, and to measure the effects of a treatment plan. If Rupert complains that he has chest pains while climbing a flight of stairs, the doctor can either change Rupert's heart medication or increase the dosage, and then ask Rupert to continue to self-collect data and visit the clinic again sometime in the future.

\subsubsection{Comparison of Situations}

In the two above scenarios, the essential presenting complaint was the same: that Rupert experienced angina after climbing a flight of stairs. Yet the decision contexts of each dramatically shaped the needs and perceptions of self-logged data in each. In the first scenario, a lack of pre-existing condition meant that the doctor started with a much larger hypothesis space, and each subsequent decision was more critical, as it could potentially affect the patient's ultimate prognosis. As a result, one would expect evidence, including self-tracked data, to be heavily scrutinized along the various dimensions in Figure 2. In the latter, the angina was viewed in the context of monitoring his already known condition. As a result, he might expect such data to be viewed in aggregate as part of larger trend, for informing his course of further treatment.

\subsection{Limitations of the Study}

The selected articles generally reflect the characteristics of research on self-tracked data in clinical settings. Although all of the selected articles were empirical and involved human subjects, they varied widely in sampling frames, population characteristics, study designs, and analytical techniques. Consequently, a meta-analysis from the selected work was not possible. Prior work in this area suggests that clinical studies of self-tracking are scarce $(2,60)$. For example, a review of pain management apps revealed that only $0.4 \%$ of apps had been evaluated for effectiveness (21). Furthermore, although there is a plethora of Quantified Self studies of self-tracking for health purposes, they have been predominantly small in scale (often with just a single participant), not peer-reviewed, and pertained to self-experimentation without clinical oversight (6). Moreover, studies involving selftracking tools are often not representative of the general population, with bias in participant selection toward computer-literate people in affluent areas (27). The general population of patients is often neither computer-literate nor affluent; older patients, for example, with chronic conditions are likely to be less able and less computer-literate [(2), p. 174], despite being a population group who would be most advantaged by the personalized medicine which self-tracking could enable (28).

A second limitation of this research is that our insights, while suggestive, are not sufficient to support any particular interventions, in terms of shaping clinical workflows to support selftracking tools, or the design of patient self-tracking tools. This highlights the importance of further empirical work and design research.

\subsection{Future Work}

The findings from this literature review provide an early overview of the challenges of using self-tracked data in clinical settings. The perspectives of clinicians from the included studies reflect that self-tracked data is a new and unfamiliar source of information. Clinicians perceived several broad themes of information quality as pertinent in deciding if and how such data could be used in decision-making. The lack of evidence from clinical trials pertaining to self-tracking tools was a significant factor in clinicians' concerns about the reliability and presentation of such data. We believe that this highlights an important area for future work: empirical studies of self-tracked data in clinical scenarios will be increasingly important, as more patients choose to engage in self-tracking practices, as these practices will inevitably manifest themselves in clinical settings.

There is the potential that self-tracking will provide new forms of detailed information useful for a future digitally enabled healthcare. Such information could improve patient outcomes, fill the gaps between consultations, and help manage long-term conditions; these uses of self-tracked data may be critical in a time where more people are suffering from chronic illness and healthcare costs are rising (6). However, this is a new research space with only limited understanding of the opportunities and challenges of self-tracked data in clinical settings. We envision that future clinical trials of self-tracking tools and practices will uncover a deeper understanding of clinicians' perceptions of quality of these forms of information. We see this as an iterative process by which clinicians (and researchers) will incrementally achieve a deeper understanding of all of the ultimate challenges to using data from self-tracking tools and practices.

\section{CONCLUSION}

In this paper, we sought to identify and understand information quality needs as they relate to the clinical use of self-logged, selftracked data. Through a literature review, we identified issues relating to accuracy and reliability, completeness, context, patient motivation, and representation. Rather than being absolutes, however, we found strong indicators to suggest that information quality needs of such data are highly dependent on the decision contexts in which data are used. In diagnosis, data are interpreted as evidence in service of particular hypotheses. Therefore, carefully interrogating self-logged data along relevant dimensions, including for individual measurements, is important to ensure proper conclusions and appropriate diagnoses are made. However, in the context of chronic care management, the data are used essentially 
to support reporting. This context is simpler, which means the space of potential misinterpretations is significantly smaller. Moreover, in the former setting, the doctor and the patient have an asymmetric relationship; while in the collaborative care management context, the two parties essentially work to enhance a shared understanding of the patient's condition and plan. In this collaborative context, some dimensions of information quality discussed, such as understanding the patient's motivations, are self-evident and simply not necessary to consider.

In summary, we believe that is significant scope for further investigation in the use of self-tracked data in clinical practice. Self-tracking practices continue to be on the rise across individuals with many different kinds of health and well-being goals, thanks, in part, to the continuing proliferation of health and activity sensing technologies as well as apps. Thus, we feel that there is an urgent need to continue to investigate information quality issues that particular clinical uses pose, as well as potential strategies relating to clinical workflows, patient self-logging practices, or the devices themselves, toward resolving such issues. In the short term, our findings support the view that, the less challenging requirements of collaborative care management may make it a more realistic first target, rather than diagnosis, for greater adoption of self-tracked data.

\section{REFERENCES}

1. International Data Corporation. IDC Forecasts Worldwide Shipments of Wearables to Surpass 200 Million in 2019, Driven by Strong Smartwatch Growth and the Emergence of Smarter Watches. (2016).

2. Neff G, Nafus D. The Self-Tracking. Cambridge: MIT Press (2016).

3. National Information Board. Personalised Health and Care 2020: Using Data and Technology to Transform Outcomes for Patients and Citizens. Technical report. London, UK (2014).

4. Swan M. The quantified self: fundamental disruption in big data science and biological discovery. Big Data (2013) 1(2):85-99. doi:10.1089/big.2012.0002

5. Dubberly H, Mehta R, Evenson S, Pangaro P. Reframing health to embrace design of our own well-being. Interactions (2010) 17(3):56-63. doi:10.1145/1744161.1744175

6. Swan M. Health 2050: the realization of personalized medicine through crowdsourcing, the quantified self, and the participatory biocitizen. J Pers Med (2012) 2(3):93-118. doi:10.3390/jpm2030093

7. Fitbit. Fitbit Second Quarter Report 2017. (2017). Available from: https:// investor.fitbit.com/overview/ir-events-and-presentations/event-details/2017/ Fitbit-Second-Quarter-2017-Financial-Results/default.aspx

8. Fausset CB, Mitzner TL, Price CE, Jones BD, Fain BW, Rogers WA. Older adults' use of and attitudes toward activity monitoring technologies. Proceedings of the Human Factors and Ergonomics Society Annual Meeting. (Vol. 57), San Diego (2013). p. 1683-7.

9. Chung C-F, CookJ, Bales E, Zia J, Munson SA. More than telemonitoring: health provider use and nonuse of life-log data in irritable bowel syndrome and weight management. J Med Internet Res (2015) 17(8):e203. doi:10.2196/jmir.4364

10. Gilleade K, Fairclough SH. Physiology as XP: body blogging to victory. BioSPlay Workshop at Fun and Games. Leuven (2010).

11. Chiauzzi E, Rodarte C, DasMahapatra P. Patient-centered activity monitoring in the self-management of chronic health conditions. BMC Med (2015) 13(1):77. doi:10.1186/s12916-015-0319-2

12. Hong MK, Wilcox L, Machado D, Olson TA, Simoneaux SF. Care partnerships: toward technology to support teens' participation in their health care. Proceedings of the 2016 CHI Conference on Human Factors in Computing Systems, CHI '16. New York, NY: ACM (2016). p. 5337-49.

13. Chung C-F, Gorm N, Shklovski IA, Munson S. Finding the right fit: understanding health tracking in workplace wellness programs. Proceedings of the

\section{AUTHOR CONTRIBUTIONS}

Substantial contributions to the conception or design of the work; or the acquisition, analysis, or interpretation of data for the work; drafting the work or revising it critically for important intellectual content; final approval of the version to be published; agreement to be accountable for all aspects of the work in ensuring that questions related to the accuracy or integrity of any part of the work are appropriately investigated and resolved: PW, MK, RG, MW, and NS. Review response and revisions: PW, MW, and MK.

\section{FUNDING}

This work was supported by the Web Science Doctoral Training Centre at the University of Southampton, and (SOCIAM: The Theory and Practice of Social Machines). The Web Science Doctoral Training Centre is part of the Research Councils UK Digital Economy Programme, funded by the Engineering and Physical Sciences Research Council (EPSRC) under grant number EP/G036926/1. The SOCIAM Project is funded by the EPSRC under grant number EP/J017728/2 and comprises the Universities of Oxford, Southampton, and Edinburgh.

2017 CHI Conference on Human Factors in Computing Systems, CHI '17. New York, NY: ACM (2017). p. 4875-86.

14. Althoff T, Sosič R, Hicks JL, King AC, Delp SL, Leskovec J. Large-scale physical activity data reveal worldwide activity inequality. Nature (2017) 547(7663):336-9. doi:10.1038/nature23018

15. Piwek L, Ellis DA, Andrews S, Joinson A. The rise of consumer health wearables: promises and barriers. PLoS Med (2016) 13(2):e1001953. doi:10.1371/ journal.pmed.1001953

16. Kim Y, Heo E, Lee H, Ji S, Choi J, Kim J-W, et al. Prescribing 10,000 steps like aspirin: designing a novel interface for data-driven medical consultations. Proceedings of the 2017 CHI Conference on Human Factors in Computing Systems, CHI '17. New York, NY: ACM (2017). p. 5787-99.

17. Karkar R, Schroeder J, Epstein DA, Pina LR, Scofield J, Fogarty J, et al. Tummytrials: a feasibility study of using self-experimentation to detect individualized food triggers. Proceedings of the 2017 CHI Conference on Human Factors in Computing Systems, CHI'17. New York, NY: ACM (2017).p. 6850-63.

18. Adams P, Murnane EL, Elfenbein M, Wethington E, Gay G. Supporting the self-management of chronic pain conditions with tailored momentary self-assessments. Proceedings of the 2017 CHI Conference on Human Factors in Computing Systems, CHI '17. New York, NY: ACM (2017). p. 1065-77.

19. Meyer J, Wasmann M, Heuten W, El Ali A, Boll SC. Identification and classification of usage patterns in long-term activity tracking. Proceedings of the 2017 CHI Conference on Human Factors in Computing Systems, CHI '17. New York, NY: ACM (2017). p. 667-78.

20. Hogan WR, Wagner MM. Accuracy of data in computer-based patient records. J Am Med Inform Assoc (1997) 4(5):342-55. doi:10.1136/jamia.1997.0040342

21. Lalloo C, Jibb LA, Rivera J, Agarwal A, Stinson JN. “There's a pain app for that": review of patient-targeted smartphone applications for pain management. Clin J Pain (2015) 31(6):557-63. doi:10.1097/AJP.0000000000000171

22. MacLeod H, Tang A, Carpendale S. Personal informatics in chronic illness management. Proceedings of Graphics Interface 2013, GI '13. Toronto, ON: Canadian Information Processing Society (2013). p. 149-56.

23. Eastwood CA, Travis L, Morgenstern TT, Donaho EK. Weight and symptom diary for self-monitoring in heart failure clinic patients. J Cardiovasc Nurs (2007) 22(5):382-9. doi:10.1097/01.JCN.0000287027.49628.a7

24. Moher D, Liberati A, Tetzlaff J, Altman DG. Preferred reporting items for systematic reviews and meta-analyses: the prisma statement. BMJ (2009) 339:b2535. doi:10.1136/bmj.b2535 
25. Katrak P, Bialocerkowski AE, Massy-Westropp N, Kumar VS, Grimmer KA. A systematic review of the content of critical appraisal tools. BMC Med Res Methodol (2004) 4(1):22. doi:10.1186/1471-2288-4-22

26. Stull DE, Leidy NK, Parasuraman B, Chassany O. Optimal recall periods for patient-reported outcomes: challenges and potential solutions. Curr Med Res Opin (2009) 25(4):929-42. doi:10.1185/03007990902774765

27. Ancker JS, Witteman HO, Hafeez B, Provencher T, Van de Graaf M, Wei E. The invisible work of personal health information management among people with multiple chronic conditions: qualitative interview study among patients and providers. J Med Internet Res (2015) 17(6):e137. doi:10.2196/jmir.4381

28. Ancker JS, Witteman HO, Hafeez B, Provencher T, Van de Graaf M, Wei E. "You get reminded you're a sick person": personal data tracking and patients with multiple chronic conditions. JMed Internet Res (2015) 17(8):e202. doi:10.2196/jmir.4209

29. Baos V, Ester F, Castellanos A, Nocea G, Caloto MT, Gerth WC, et al. Use of a structured migraine diary improves patient and physician communication about migraine disability and treatment outcomes. Int J Clin Pract (2005) 59(3):281-6. doi:10.1111/j.1742-1241.2005.00469.x

30. Choo S, Kim JY, Jung SY, Kim S, Kim JE, Han JS, et al. Development of a weight loss mobile app linked with an accelerometer for use in the clinic: usability, acceptability, and early testing of its impact on the patient-doctor relationship. JMIR Mhealth Uhealth (2016) 4(1):e24. doi:10.2196/mhealth.4546

31. Chung C-F, Dew K, Cole A, Zia J, Fogarty J, Kientz JA, et al. Boundary negotiating artifacts in personal informatics: patient-provider collaboration with patient-generated data. Proceedings of the 19th ACM Conference on ComputerSupported Cooperative Work \& Social Computing, CSCW'16. New York, NY: ACM (2016). p. 770-86.

32. Schroeder J, Hoffswell J, Chung C-F, Fogarty J, Munson S, Zia J. Supporting patient-provider collaboration to identify individual triggers using food and symptom journals. CSCW Conf Comput Support Coop Work (2017) 2017:1726-39. doi:10.1145/2998181.2998276

33. Cohen DJ, Keller SR, Hayes GR, Dorr DA, Ash JS, Sittig DF. Integrating patient-generated health data into clinical care settings or clinical decision-making: lessons learned from project healthdesign. JMIR Hum Factors (2016) 3(2):e26. doi:10.2196/humanfactors.5919

34. Friess M, Stukenberg E. A quantitative pilot study on the use of a fitness tracker in the preventative management of employees at risk of chronic disease in a health care facility. Online J Nurs Inform (2016) 19(3):1.

35. Huba N, Zhang Y. Designing patient-centered personal health records (PHRs): health care professionals' perspective on patient-generated data. J Med Syst (2012) 36(6):3893-905. doi:10.1007/s10916-012-9861-z

36. Jacobs ML, Clawson J, Mynatt ED. My journey compass: a preliminary investigation of a mobile tool for cancer patients. Proceedings of the SIGCHI Conference on Human Factors in Computing Systems, CHI '14. New York, NY: ACM (2014). p. 663-72.

37. Kim Y, Ji S, Lee H, Kim J-W, Yoo S, Lee J. "My doctor is keeping an eye on me!" Exploring the clinical applicability of a mobile food logger. Proceedings of the 2016 CHI Conference on Human Factors in Computing Systems, CHI '16. New York, NY: ACM (2016). p. 5620-31.

38. Mishra SR, Haldar S, Pollack AH, Kendall L, Miller AD, Khelifi M, et al. "Not just a receiver": understanding patient behavior in the hospital environment. Proceedings of the 2016 CHI Conference on Human Factors in Computing Systems, CHI '16. New York, NY: ACM (2016). p. 3103-14.

39. Nundy S, Lu C-YE, Hogan P, Mishra A, Peek ME. Using patient-generated health data from mobile technologies for diabetes self-management support: provider perspectives from an academic medical center. J Diabetes Sci Technol (2014) 8(1):74-82. doi:10.1177/1932296813511727

40. Patel RA, Klasnja P, Hartzler A, Unruh KT, Pratt W. Probing the benefits of real-time tracking during cancer care. AMIA Annual Symposium Proceedings. (Vol. 2012) (2012). p. 1340-9.

41. Piras EM, Miele F. Clinical self-tracking and monitoring technologies: negotiations in the ICT-mediated patient-provider relationship. Health Sociol Rev (2017) 26(1):38-53. doi:10.1080/14461242.2016.1212316

42. Steward DA, Hofler RA, Thaldorf C, Milov DE. A method for understanding some consequences of bringing patient-generated data into health care delivery. Med Decis Making (2010) 30(4):E1-13. doi:10.1177/0272989X10371829

43. Vandenberghe B, Geerts D. Sleep monitoring tools at home and in the hospital: bridging quantified self and clinical sleep research. Proceedings of 9th
International Conference on Pervasive Computing Technologies for Healthcare. Istanbul: ICST (2015).

44. West P, Giordano R, Van Kleek M, Shadbolt N. The quantified patient in the doctor's office: challenges \& opportunities. Proceedings of the $2016 \mathrm{CHI}$ Conference on Human Factors in Computing Systems, CHI '16. New York, NY: ACM (2016). p. 3066-78.

45. Zhu H, Colgan J, Reddy M, Choe EK. Sharing patient-generated data in clinical practices: an interview study. AMIA Annu Symp Proc (2016) 2016:1303-12.

46. Zia JK, Chung C-F, Schroeder J, Munson SA, Kientz JA, Fogarty J, et al. The feasibility, usability, and clinical utility of traditional paper food and symptom journals for patients with irritable bowel syndrome. Neurogastroenterol Motil (2016) 29(2):e12935. doi:10.1111/nmo.12935

47. Patel R, Sandars J, Carr S. Clinical diagnostic decision-making in real life contexts: a trans-theoretical approach for teaching: AMEE Guide No. 95. Med Teach (2014) 37:211-27. doi:10.3109/0142159X.2014.975195

48. Krefting L. Rigor in qualitative research: the assessment of trustworthiness. Am J Occup Ther (1991) 45(3):214-22. doi:10.5014/ajot.45.3.214

49. Leininger MM. Nature, rationale, and importance of qualitative research methods in nursing. In: Leininger MM, editor. Qualitative Research Methods in Nursing. Orlando, FL: Grune \& Stratton (1985). p. 1-25.

50. Agar M. Speaking of Ethnography. Newbury Park: SAGE (1986). 2 p.

51. Zhu H, Colgan J, Reddy M, Choe EK. Sharing patient-generated data in clinical practices: an interview study. AMIA Annual Symposium Proceedings. (Vol. 2016), Chicago: American Medical Informatics Association (2016). 1303 p.

52. Olfson M, Mechanic D, Hansell S, Boyer CA, Walkup J, Weiden PJ. Predicting medication noncompliance after hospital discharge among patients with schizophrenia. Psychiatr Serv (2000) 51(2):216-22. doi:10.1176/appi. ps.51.2.216

53. Krahn LE, Li H, O'Connor MK. Patients who strive to be ill: factitious disorder with physical symptoms. Am J Psychiatry (2003) 160(6):1163-8. doi:10.1176/ appi.ajp.160.6.1163

54. Mann RS. Differential diagnosis and classification of apathy. Am J Psychiatry (1990) 147(1):22-30. doi:10.1176/ajp.147.1.22

55. Choe EK, Lee NB, Lee B, Pratt W, Kientz JA. Understanding quantified-selfers' practices in collecting and exploring personal data. Proceedings of the SIGCHI Conference on Human Factors in Computing Systems, CHI '14. New York, NY: ACM (2014). p. 1143-52.

56. Jones SL. Exploring correlational information in aggregated quantified self data dashboards. Proceedings of the 2015 ACM International Joint Conference on Pervasive and Ubiquitous Computing and Proceedings of the 2015 ACM International Symposium on Wearable Computers, UbiComp '15. New York, NY: ACM (2015). p. 1075-80.

57. Rapp A, Cena F. Self-monitoring and technology: challenges and open issues in personal informatics. In: Stephanidis C, Antona M, editors. Universal Access in Human-Computer Interaction. Design for All and Accessibility Practice, Number 8516 in Lecture Notes in Computer Science. Heraklion: Springer (2014). p. 613-22.

58. Woods SS, Evans NC, Frisbee KL. Integrating patient voices into health information for self-care and patient-clinician partnerships: veterans affairs design recommendations for patient-generated data applications. J Am Med Inform Assoc (2016) 23(3):491-5. doi:10.1093/jamia/ocv199

59. Third A, Gkotsis G, Kaldoudi E, Drosatos G, Portokallidis N, Roumeliotis S, et al. Integrating medical scientific knowledge with the semantically quantified self. International Semantic Web Conference. Kobe: Springer (2016). p. 566-80.

60. Payne HE, Lister C, West JH, Bernhardt JM. Behavioral functionality of mobile apps in health interventions: a systematic review of the literature. JMIR Mhealth Uhealth (2015) 3(1):e20. doi:10.2196/mhealth.3335

Conflict of Interest Statement: The authors declare that the research was conducted in the absence of any commercial or financial relationships that could be construed as a potential conflict of interest.

Copyright $\odot 2017$ West, Van Kleek, Giordano, Weal and Shadbolt. This is an openaccess article distributed under the terms of the Creative Commons Attribution License (CC BY). The use, distribution or reproduction in other forums is permitted, provided the original author(s) or licensor are credited and that the original publication in this journal is cited, in accordance with accepted academic practice. No use, distribution or reproduction is permitted which does not comply with these terms. 